\title{
La gestión contable como elemento determinante en el desarrollo sostenible de las Mipymes
}

Accounting management as a determining element in the sustainable development of Mipymes

Karina Johanna Iza López. ${ }^{1}$

\begin{abstract}
DOI: https://doi.org/10.33262/concienciadigital.v4i4.1.1923

Introduction, MSMEs are the main business fabric in the world, these organizations are the product of the existence of a business idea that starts from the need to satisfy something and is undoubtedly materialized with the work of whoever creates them, such as the entrepreneur. According to the legislation of each country where they are located, MSMEs are organizations that are classified by some criteria, one of the main ones is their size, which allows them to be grouped into micro, small and medium-sized companies, which, although they represent a basic pillar in the economies of the countries in which they put their business mission into practice have limitations, one of which is the lack of accounting management, which makes it easier for them to satisfy the variety of people's requirements without affecting the needs of future generations. Objective. Determine if accounting management is a determining element in the sustainable development of MSMEs. Methodology. The type, focus, and scope were nonexperimental, qualitative-quantitative, and descriptive. The population taken for the analysis was 969 MSMEs from the Guaranda, from this number a sample of 369 organizations emerged. The information was the result of the application of the survey

1 Universidad Estatal de Bolívar, Facultad de Ciencias Administrativas, Gestión Empresarial e Informática, Carrera de Contabilidad y Auditoría, Guaranda, Ecuador, kiza@ueb.edu.ec, https://orcid.org/0000-0002-5111-8792.
\end{abstract}


and the questionnaire, the latter was validated in relation to the reliability of the questions through the collaboration of 5 highly prestigious experts. Results. The owners of MSMEs do not have basic notions about accounting, but they are aware that accounting management is a determining element to bring out essential business skills to achieve a balance between the economic, social and environmental contexts. Conclusion. The scarce accounting knowledge has meant that managers do not reveal elements inherent to sustainable development.

Keywords: entrepreneurship, accounting management, MSMEs, sustainable development.

\section{Resumen}

Introducción, las Mipymes son el principal tejido empresarial en el mundo, estas organizaciones son producto de la existencia de una idea de negocio que parte de la necesidad de satisfacer algo y se materializa sin lugar a dudas con el trabajo de quien las crea como es el emprendedor. Las Mipymes de acuerdo a la legislación de cada país donde se ubican son organizaciones que se clasifican por algunos criterios, uno de los principales es el tamaño lo cual permite agruparlas en micro, pequeñas y medianas empresas, que, aunque representan un pilar básico en la economía de los países en los que ponen en práctica su misión empresarial tienen limitaciones una de ellas la falta de una gestión contable, que les facilite satisfacer la variedad de requerimientos de las personas sin afectar aquellas necesidades de las generaciones venideras. Objetivo. Determinar si la gestión contable es un elemento determinante en el desarrollo sostenible de las Mipymes. Metodología. El tipo, el enfoque y el alcance fue no experimental, cualicuantitativo, y descriptivo. La población tomada para el análisis fueron 969 Mipymes del cantón Guaranda, de este número se desprendió una muestra de 369 organizaciones. La información fue resultado de la aplicación de la encuesta y el cuestionario, este último fue validado en relación a la confiabilidad de las preguntas mediante la colaboración de 5 expertos de gran prestigio. Resultados. Los propietarios de las Mipymes no tienen nociones básicas sobre la contabilidad, pero son conscientes que la gestión contable es un elemento determinante para sacar a relucir capacidades empresariales esenciales para lograr un equilibrio entre los contextos económico, social y ambiental. Conclusión. El escaso conocimiento contable ha conllevado a que los gestores no pongan de manifiesto elementos inherentes al desarrollo sostenible.

Palabras claves: emprendimiento, gestión contable, Mipymes, desarrollo sostenible.

\section{Introducción}

Las Mipymes por su forma de hacer negocios en el contexto empresarial en la actualidad están siendo reconocidas como el pilar básico que dinamiza la economía global del mundo, entendiendo que su labor se centra en empresas que están figuradas en el mercado por criterios como el tamaño lo cual hace que aborden la labor empresarial desde el tejido pequeño, grande y mediano, el capital que es limitado, el número de empleados, estructura 
organizacional entre otros. La tipificación empresarial de estas organizaciones depende en gran medida de la legislación del país en el que se ubiquen.

Este tipo de negocios tienen sus propias peculiaridades que las muestran en la atención que brindan a las personas para satisfacer sus necesidades. En el caso del Ecuador las Mipymes son la principal fuente generadora de empleo por estar inmersas en todas las diligencias económicas como, por ejemplo: la comercialización, la producción, distribución, financiamiento, entre otras.

Las Mipymes son organizaciones que pertenecen a la economía popular y solidaria que como se indicó en líneas anteriores son pieza importante de la economía del país independiente de su naturaleza económica, no obstante, presentan algunas limitantes como el capital, fuentes de fondeo escasas, infraestructura física limitada, sus gestores no cuentan con nociones básicas de contabilidad que les permitan tomar decisiones para un mejor desempeño sostenible, entre otros.

En este marco de ideas, el objetivo del presente artículo científico es determinar si la gestión contable es un factor determinante en el desarrollo sostenible de las Mipymes.

Los emprendimientos instituidos en los distintos países del mundo son producto del deseo, del esfuerzo, de la dedicación, necesidad, de la idea de negocio, el compromiso, entre otros del emprendedor lo cual da a lugar a señalar que para integrarse como tal no existió un estudio técnico de por medio, peor aún de la combinación de elementos como la tecnología de la información y comunicación (TIC), recursos suficientes, áreas de conocimiento como administración, contabilidad, finanzas, marketing, entre otras áreas que deben conocerse para elaborar y disponer de herramientas efectivas de gestión (Pereira-Bolaños, 2019). Los emprendimientos al traducirse en Mipymes; es decir, en micro, pequeñas y medianas empresas pese a que juegan un rol protagónico en la matriz de producción son los entes más vulnerables puesto que sus condiciones de constitución tienden a durar poco tiempo en el mercado; esto se debe a que disponen de algunas limitaciones como las expuestas en líneas anteriores sobre todo en el área de conocimiento denominada contabilidad lo que minimiza su accionar en el desarrollo sostenible (MiteAlbán, 2018) .

En la actualidad las Mipymes son entendidas como aquellas organizaciones vulnerables que luchan a diario para perdurar en el mercado global pese a sus limitaciones y a partir de lo expuesto a nacido la necesidad de estudiar elementos determinantes en el éxito de las mismas. No obstante, existen también aquellos elementos que de una u otra manera inhiben el quehacer de negocios que son conocidos como "factores limitantes", entre uno de las principales es la escasa o nula formación empresarial de los gestores de empresas relacionado con la sabiduría de un sinnúmero de ciencias una de ellas la contabilidad (Alva, 2017).

En términos generales la contabilidad es la disciplina que se encarga de brindar información del patrimonio y la liquidez de las empresas aunque en el caso de las micro, pequeñas y medianas que integran un grupo heterogéneo que representan el más 
importante tejido empresarial que gestiona su misión empresarial bajo el marco de la autonomía; tal es así, que no están obligadas a rendir cuentas ni a publicar estados financieros para nadie en absoluto (Gómez-Villegas, 2016). Por otro lado, si bien es cierto las Mipymes no están obligadas a publicar para uso externo sus informes contables, si se hace necesario que dispongan de un modelo de gestión para satisfacer sus necesidades de información de uso interno en miras de conocer su progreso en un periodo contable para mejorar o fortalecer su misión y de esta manera garantizar un desarrollo sostenible apropiado (Delgado \& Gómez-Chi, 2019).

La contabilidad está presente en el quehacer de negocios desde que el hombre empezó a dar paso a las diligencias de tipo mercantil; debido que a partir de las cuáles surgió la necesidad de conocer los resultados obtenidos mediante el control de los hechos económicos visto en lo que ingresa y sale en términos monetarios (Escobar, 2016). En el caso particular de las empresas independiente a su naturaleza para enmarcarse en la legislación contable como políticas, procesos, procedimientos, NIC, NIIF. NIIF para Pymes, entre otros el emprendedor, el empresario, el gestor debe tener claro que debe tener conocimiento sobre postulados contables lo cual verá reflejado en los resultados alcanzados mismo que están registrados en los Estados Financieros (Rodríguez-Guzmán, 2014).

En este sentido, la contabilidad es la ciencia encargada de identificar, figurar, registrar, entre otros las transacciones de las organizaciones apoyada en fuentes de información válida que sigue un proceso para emitir los Estados Financieros alineados a los factores: económico, social y ambiental que facilita el conocimiento a detalle de los logros o desaciertos de las empresas (Saavedra-García, 2015; Morales-Espinoza \& MoralesEspinosa, 2019).

El desarrollo sostenible son las practicas empleadas por las empresas para dar lugar a su misión empresarial desde tres ámbitos como son: el progreso económico, pero sin dejar de lado la parte social y la gestión ambiental, pero esto lo hace con conocimiento de causa sobre los resultados obtenidos y bajo el marco legal (Miranda et al., 2007). Este criterio concuerda con lo manifestado por los autores Chirinos et al. (2017), que indican que el emprendimiento reflejado en las micro, pequeñas y medianas empresas es visto como el resultado de la implementación de un conjunto de sanas prácticas con el propósito de lograr lo planeado mediante la satisfacción de necesidades sin dañar su entorno. En líneas generales, es una forma de organización que permite el mejor uso de los recursos para el progreso económico traducido en fuentes de empleo, beneficios económicos en el marco de una competencia leal, el progreso social mediante la satisfacción de necesidades con bienes o servicios de alta calidad, aportando con el desarrollo del espacio donde opera y la gestión ambiental mediante su cuidado y protección (Rodríguez-Moreno, 2016; Chirinos-Araque \& Meriño-Cordoba, Emprendimiento sostenible como estrategia para la creación de empresas, 2018).

La gestión contable a cualquier empresa proporciona basta información a sus gestores para la toma de decisiones puesto que se instituye en una herramienta de control de los 
negocios, en otras palabras es el termómetro que posibilita medir el grado de despeño empresarial debido a que integra todo un proceso que parte de la identificación de los documentos mercantiles negociables y no negociables utilizados de respaldo contable, el registro de las transacciones iniciales mediante el estado de situación financiera, la jornalización de la carga transaccional, la mayorización, el cotejo minucioso de saldos, la hoja de trabajo, los asientos de ajuste y cierre y los estados financieros .

Por lo general las empresas crean y utilizan la información contable conforme el marco jurídico relacionado como las NIC, las NIIF, NIIF para pymes, entre otras para saciar las necesidades de los dos tipos de usuarios que dispone como son los internos y externos, pues a estos les permite medir el desempeño de negocios en distintos contextos uno de ellos el progreso sostenible (Sánchez-Esrella \& Tarodo-Pisonero, 2015; García-Carvajal, 2016). En consecuencia, también es menester indicar que, para el caso específico de las micros, pequeñas y medianas empresas las NIIF para Pymes es de carácter obligatorio.

La ciencia contable al ser práctica es de suma importancia para las organizaciones por dos razones en especial: 1) mantiene registros generados con periodicidad diaria; y, 2) proporciona amplia información de la empresa mediante los estados financieros esto le ayuda a mantenerse operando en el mercado (Montoya-Fuentes \& Flores-Zeledón, 2015; Villalba-Hidalgo \& Zambrano-Macías, 2016).

En síntesis, la gestión contable es básica para el control de todas las transacciones generadas en la empresa que es resultado de la pronta respuesta a las necesidades del mercado global, lo cual les posibilita abrir el abanico de oportunidades respecto a los diferentes tipos de desarrollo uno de ellos el sostenible.

\section{Metodología}

Para dar lugar el objetivo propuesto en el artículo científico como es determinar si la gestión contable es un elemento determinante del desarrollo sostenible en la Mipymes del cantón Guaranda se consideró el tipo de la investigación mixta; es decir, cualitativa y cuantitativa porque se maniobró datos de tipo no numérica y numérica, está última al procesar la información generada, el enfoque no experimental puesto que los datos se tomaron de las Mipymes de manera espontánea mediante la observación sin manipulación alguna, el alcance descriptivo porque se estableció aspectos sobre las peculiaridades de las micro, pequeñas y medianas empresas (Mipymes) y sus gestores, información sobre la gestión contable. La población fue de 969 micros, pequeñas y medianas empresas de la cual se tomó como muestra 369 negocios de los sectores urbano y rural. Los datos se recopilaron mediante una encuesta debidamente estructurada con un cuestionario validado por expertos de prestigio de la Universidad Estatal de Bolívar. La información se procesó con el soporte del programa informático SPSS 20.0.

\section{Resultados}

El 100\% de las Mipymes en el cantón Guaranda son de tamaño micro y pequeñas puesto que disponen de 1 hasta 49 empleados. El 95\% de los negocios ostentan una estructura 
de tipo familiar lo que denota que cualquier decisión por más simple que sea es tomada por quienes integran la familia lo cual hace que exista concentración en las decisiones y apenas un 5\% tiene una estructura distinta. El 100\% de los emprendimientos están dedicados a atender las necesidades de las personas en el mercado que cada vez es más exigente desde el horizonte del comercio y servicios. Este tipo de empresas que en los últimos tiempos se han convertido en el eje principal de la economía del país en el caso del cantón Guaranda en un $80 \%$ de negocios perduran en el mercado de 0 a 1 año, el $17 \%$ de 2 a 5 años y el 3\% mayor o igual a 5 años. Respecto a la fuente de financiamiento se desplegó que el 85\%,10\% y 5\% de las Mipymes en ese orden cristalizaron la idea de negocio con recursos que nacieron de la familia, instituciones financieras independiente a si se trata de un Banco o una Cooperativas de Ahorro y Crédito de la localidad y fondos propios producto de ahorros, como se muestra en la tabla 1.

\section{Tabla 1}

Peculiaridades de las Mipymes

\begin{tabular}{|c|c|}
\hline Variables & Porcentaje \\
\hline \multicolumn{2}{|c|}{ Tamaño empresarial de acuerdo al número de empleados. } \\
\hline De 1 a 9 colaboradores & $95 \%$ \\
\hline De 10 a 49 colaboradores & $5 \%$ \\
\hline De 50 a 199 colaboradores & $0 \%$ \\
\hline$\geq 200$ colaboradores & $0 \%$ \\
\hline \multicolumn{2}{|l|}{ Estructura Organizacional } \\
\hline Familiar & $95 \%$ \\
\hline Otra & $5 \%$ \\
\hline \multicolumn{2}{|l|}{ Diligencia económica } \\
\hline Industrial & $0 \%$ \\
\hline Comercial & $85 \%$ \\
\hline Servicios & $15 \%$ \\
\hline \multicolumn{2}{|l|}{ Presencia en el mercado } \\
\hline De 0 a 1 año & $80 \%$ \\
\hline De 2 a 5 años & $17 \%$ \\
\hline$>=5$ años & $3 \%$ \\
\hline \multicolumn{2}{|l|}{ Financiamiento } \\
\hline Familia & $85 \%$ \\
\hline Fondos propios & $5 \%$ \\
\hline IFI'S & $10 \%$ \\
\hline Otras & $0 \%$ \\
\hline
\end{tabular}

Fuente: Elaboración propia.

La edad de los emprendedores que pusieron todo su esfuerzo, dedicación y adeudo para la creación de sus negocios se concentra en un $40 \%, 35 \%, 15 \%$ y $10 \%$ en ese orden en los rangos: de 30 a 40, 41 a 50, igual o mayor a 20 a 51 años. El deseo de emprender de 
acuerdo al análisis realizado se concentra en un $70 \%$ en mujeres esto por la necesidad principal de soportar económicamente a los integrantes de su núcleo familiar y el $30 \%$ restante en hombres. En lo que se relaciona al grado de formación el $80 \%$ se enmarca en primaria y secundaria y el $20 \%$ restante entre pregrado, posgrado y ninguna formación. Pese a que los emprendimientos están instituidos el 95\% de los emprendedores no tienen formación empresarial que soporte la labor de negocios; tan solo el 5\% si cuenta con formación en áreas afines a administración de empresas, aspecto que va de la mano con la experiencia en el mundo empresarial, como se muestra en la tabla 2.

Tabla 2

Peculiaridades de las Mipymes

\begin{tabular}{ll}
\hline \multicolumn{1}{c}{ Variables } & Porcentaje \\
\hline Rango de edad & \\
$\geq 20$ años & $15 \%$ \\
De 30 a 40 años & $40 \%$ \\
De 41 a 50 años & $35 \%$ \\
$\geq 51$ años & $10 \%$ \\
Género & \\
Femenino & $70 \%$ \\
Masculino & $30 \%$ \\
Grado de educación & \\
Primaria & $40 \%$ \\
Secundaria & $40 \%$ \\
Pregrado & $15 \%$ \\
Posgrado & $4 \%$ \\
Ninguna & $1 \%$ \\
Formación en el contexto empresarial & \\
Sí & $5 \%$ \\
No & $95 \%$ \\
Experiencia en el ámbito del emprendimiento & \\
Sí & $5 \%$ \\
No & $95 \%$ \\
\hline
\end{tabular}

Fuente: Elaboración propia

El 95\% de los emprendedores no disponen de nociones básicas sobre la disciplina denominada contabilidad y aquellos que disponen representan un número reducido que se enmarca en el 5\% quienes se han formado en áreas como marketing, administración de empresas, contabilidad, auditoría, finanzas, entre otras. En la línea del desarrollo del proceso contable apenas el 5\% lo hace de manera manual apenas con la utilización de una hoja de Microsoft Excel en el mejor de los casos en otras mediante la asistencia de un cuaderno contable. Los gestores de negocios pese a que no disponen del conocimiento contable, en su totalidad manifestaron lo importante que es conocer los resultados 
generados y que para esto necesitan de manera imperiosa los estados financieros que a la final se constituyen en documentos que les ayudarán a tomar decisiones, pero para llegar hasta este punto requieren un modelo de gestión que sirva como guía y facilite la puesta en práctica de acciones sanas desde lo económico, social y ambiental para lograr el desarrollo sostenible deseado para subsistir en el mercado, como se muestra en la tabla 3.

Tabla 3

Peculiaridades de las Mipymes

\begin{tabular}{ll}
\hline \multicolumn{1}{c}{ Variables } & Porcentaje \\
\hline Fundamentos de contabilidad & $5 \%$ \\
Sí & $95 \%$ \\
No & \\
Manejo de contabilidad & \\
Sí & $5 \%$ \\
No & $95 \%$ \\
Tipo de contabilidad. & \\
Manual & $5 \%$ \\
Automatizada & $0 \%$ \\
Ninguna & $95 \%$ \\
Reportes Financieros en la toma de decisiones. & \\
Si & $100 \%$ \\
No & $0 \%$ \\
Modelo de gestión y desarrollo sostenible. & \\
Si & $100 \%$ \\
No & $0 \%$ \\
\hline
\end{tabular}

Fuente: Elaboración propia.

\section{Conclusiones}

- Los negocios son dirigidos en gran parte por mujeres luchadoras que ostentas edades maduras que están entre los 30 años en adelante que desean aportar con un granito de arena en el progreso económico personal y familiar puesto que tienen la necesidad de satisfacer las principales necesidades vitales de los integrantes de su núcleo familiar; sin embargo, no tienen la formación empresarial necesaria puesto que su formación de educación apenas se centra en la primaria y secundaria.

- La gran mayoría de los negocios son micros y pequeñas debido a que se valoró el número de empleados que laboran, mismas que se dedican a establecer diligencias de comercio y a la prestación de servicios, pero presentan debilidades como el escaso financiamiento, los recursos que disponen son limitados; tal es así que para integrarse como ente económico utilizaron 
recursos nacientes principalmente de recursos propios y muy pocas de estas organizaciones se han financiado de instituciones financieras de la localidad

- El deseo, el compromiso de los emprendedores está latente; sin embargo, no existe en su gran mayoría el conocimiento sobre postulados contables lo que de cierta maneta limita el quehacer de las micro, pequeñas, mediana empresas en relación a generar e implementar herramientas de gestión como un modelo de gestión contable que les facilite la toma de decisiones en la aplicación de buenas prácticas para lograr un desarrollo empresarial sostenible.

\section{Referencias bibliográficas}

Alva, E. (2017). La desaparición de las microempresas en el Perú. Una aproximación a los factores que predisponen a su mortalidad. Caso del Cercado de Lima. Economía y Desarrollo, 158(2), 76-90.

Chirinos-Araque, Y., \& Meriño-Cordoba, H. (2018). Emprendimiento sostenible como estrategia para la creación de empresas. Science of Human Action, 3(1), 11-14. doi:https://doi.org/10.21501/2500-669X.2707

Chirinos-Araque, Y., Meriño-Cordoba, V. H., Martínez-Meriño, C. I., \& Pérez-Peralta, C. M. (2017). El emprendimiento sostenible como generador de conocimiento en las PYMES. Gestión del Conocimiento Perspectiva Multidisiplinaria., 39(7), 3658.

Delgado, G. I., \& Gómez-Chi, S. V. (2019). Importancia de la contabilidaddentro de las Pymes. Contribuciones a la Economía , 1-13. Obtenido de hdl.handle.net/20.500.11763/ce193contabilidad-pymes

Escobar, M. E. (2016). Historia de la Contabilidad. Apuntes Contables, 17, 55 - 176.

García-Carvajal, S. \&.-C. (2016). Los aspectos cualitativos en las Pymes y los nuevos retos gerenciales frente a las NIIF. Academia y Virtualidad, 9(2).

Gómez-Villegas, M. (2016). NIIF y MIPYMES: retos de la contabilidad para el contexto y la productividad. Cuadernos de Administración, 29(53), 49-76.

Miranda, T., Suset, A., Cruz, A., Machado , H., \& Campos, M. (2007). El Desarrollo sostenible: Perspectivas y enfoques en una nueva época. Pastos y Forrajes [online], 30(2), $\quad 1-5 . \quad$ Obtenido de http://scielo.sld.cu/scielo.php?script=sci_arttext\&pid=S0864-039420070

Mite-Albán, M. T. (2018). Estrategias de contabilidad de gestión aplicada a pymes revisión literaria. Lasallista de investigación, 15(2), $256 \quad-270$. doi:doi:https://doi.org/10.22507/rli.v15n2a20 
Montoya-Fuentes, L. P., \& Flores-Zeledón, Y. M. (2015). Diseño e implementación de un sistema contable en la empresa COSMOS SERVICENTER AUTOLAVADO de la ciudad de Estelí durante el mes de Octubre del año 2014. [Tesis de pregrado, UNAN-MANAGUA] Re-UNAN-MANAGUA.

Morales-Espinoza, D. Y., \& Morales-Espinosa, J. M. (2019). La contabilidad un elemento indispensable en el desarrollo de las empresas. 3(2), 49-68. doi:https://doi.org/10.33970/eetes.v3.n2.2019.136

Pereira-Bolaños, C. C. (2019). Actualidad de la gestión empresarial en las pymes. Apuntes Contables, 24, 39-53. doi:doi:https://doi.org/10.18601/16577175.n24.03.

Rodríguez-Guzmán, D. C. (2014). Cómo afectará la aplicación de las normas internacionales de contabilidad a las pymes en Colombia. Apuntes Contables, 17, 59-110.

Rodríguez-Moreno, D. C. (2016). Emprendimiento sostenible, significado y dimensiones. Katharsis, 21, 419-448.

Saavedra-García, M. L.-G. (2015). La investigación contable en Latinoamérica. Actualidad Contable Faces, 18(31), 99-121.

Sánchez-Esrella, O. C., \& Tarodo-Pisonero, C. (2015). Gestión contable. España: Paraninfo.

Villalba-Hidalgo, D. I., \& Zambrano-Macías, M. J. (2016). Propuesta de un sistema contable para optimizar la rentabilidad del ITSG. [Tesis de pregrado, UG ] Re$U G$.

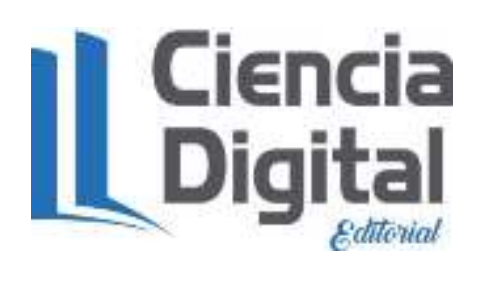




\section{PARA CITAR EL ARTÍCULO INDEXADO.}

Iza López, K. J. (2021). La gestión contable como elemento determinante en el desarrollo sostenible de las Mipymes . ConcienciaDigital, 4(4.1), 39-49. https://doi.org/10.33262/concienciadigital.v4i4.1.1923

\section{Ciencia \\ Ligital \\ Edhorial}

El artículo que se publica es de exclusiva responsabilidad de los autores y no necesariamente reflejan el pensamiento de la Revista Conciencia Digital.

El artículo queda en propiedad de la revista y, por tanto, su publicación parcial y/o total en otro medio tiene que ser autorizado por el director de la Revista Conciencia Digital.

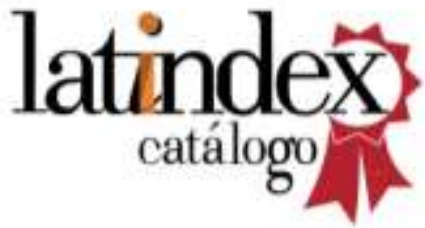

